ACCEPted For ApJ Letters 2014 MAy 29

Preprint typeset using $\mathrm{LT}_{\mathrm{E}} \mathrm{X}$ style emulateapj v. 5/2/11

\title{
RESONANCES OF MULTIPLE EXOPLANETS AND IMPLICATIONS FOR THEIR FORMATION
}

\author{
Xiaojia Zhang ${ }^{1 *}$, Hui Li $^{2}$, Shengtai Li ${ }^{2}$, Douglas N. C. $\operatorname{Lin}^{1,3}$ \\ Accepted for ApJ Letters 2014 May 29
}

\begin{abstract}
Among 160 of the multiple exoplanetary systems confirmed, about $30 \%$ of them have neighboring pairs with a period ratio $\leq 2$. A significant fraction of these pairs are around mean motion resonance (MMR), more interestingly, peak around 2:1 and 3:2, with a clear absence of more closely packed MMRs with period ratios less than 4:3, regardless of planet masses. Here we report numerical simulations demonstrating that such MMR behavior places important constraints on the disk evolution stage out of which the observed planets formed. Multiple massive planets (with mass $\geq 0.8 M_{\text {Jup }}$ ) tend to end up with a 2:1 MMR mostly independent of the disk masses but low-mass planets (with mass $\left.\leq 30 M_{\oplus}\right)$ can have MMRs larger than 4:3 only when the disk mass is quite small, suggesting that the observed dynamical architecture of most low-mass-planet pairs was established late in the disk evolution stage, just before it was dispersed completely.

Subject headings: planet-disk interactions - planetary systems - protoplanetary disks
\end{abstract}

\section{INTRODUCTION}

Many multiple planetary candidates were discovered by Kepler's transit search. It has been pervasively suggested that the majority of them are indeed genuine multiple-planet systems (Lissauer et al. 2012; Batalha et al. 2013). A significant fraction of adjacent pairs of planets are in or near mean motion resonances (MMRs) with period ratios around 2:1, 3:2, 5:3, etc. (Lissauer et al. 2011b), although members of most multiple systems do not have nearly commensurable orbits (Mayor et al. 2009).

The MMR of exoplanet systems has been well studied under various perspectives (Goldreich 1965; Wisdom 1980; Henrard 1982; Weidenschilling \& Davis 1985; Ogihara \& Ida 2009; Ogihara et al. 2010; Lee et al. 2013). A widely adopted scenario is that resonant pairs captured each other on their mutual MMRs through convergent migration (Bryden et al. 2000; Kley 2000; Lee \& Peale $2002 b)$. Migration mechanisms include tidal interaction between very short period planets and their host stars (Schlaufman et al. 2010) as well as protoplanet interaction with their natal disks (Lin et al. 1996). For planets with periods longer than a few days, MMRs provide supporting evidence of the disk migration scenario.

Gas-giant planets with sufficient mass to open gaps in their natal disks undergo Type II migration (Lin \& Papaloizou 1986). Most Kepler candidates have much lower masses and undergo Type I migration (Goldreich \& Tremaine 1980; Ward 1997; Paardekooper et al. 2011; Kretke \& Lin 2012). In either case, orbital convergence would occur if the inward migration of a planet catches up with that of its siblings closer to the host star or if a planet is trapped at some special disk region and its siblings migrate toward it. Convergent migration leads

\footnotetext{
*E-mail: xzhang47@ucsc.edu

${ }^{1}$ Department of Astronomy and Astrophysics, University of California, Santa Cruz, CA 95064, USA

${ }^{2}$ Los Alamos National Laboratory, Los Alamos, NM 87545, USA

${ }^{3}$ Institute for Advanced Studies, Tsinghua University, Beijing, China
}

to the possibility of resonant capture (Lee \& Peale 2002a; Kley et al. 2004).

Many multiple planet systems are indeed locked in or are close to MMRs. The current database on The Extrasolar Planets Encyclopaedia Website (http://exoplanet.eu) contains 157 confirmed multiple planet systems with $\sim 225$ neighboring pairs. This sample includes 118 systems of 2 planets, 22 systems of 3 planets, 9 systems of 4 planets, 4 systems of 5 planets and 4 systems of 6 planets. Figure 1 shows the distribution of these adjacent pairs as a function of their period ratios, focusing on systems with a period ratio around and less than 4 . Overall, $\sim 70$ pairs (i.e., $\sim 1 / 3$ of all pairs) have a period ratio around or less than 2 . The subsets of pairs with both planets' masses $>0.8 M_{\text {Jupiter }}$ and below $\sim 30 M_{\oplus}$ (designated as gas-giant and low-mass-planet systems respectively) are also shown in Figure 1. Most gas giants are discovered by the radial velocity method. Although their mass determinations are lower limits, this observational bias does not affect their statistics. Most multiple low-mass-planet systems are Kepler candidates. Their mass has been estimated by using an empirical formula to convert a planet's radius to mass (Lissauer et al. 2011b,a).

Figure 1 confirms the previously known results that there are statistically clusters of systems in or near the 2:1 and 3:2 MMRs (Lissauer et al. 2011b; Fabrycky \& Kepler Science Team 2012). Since the confirmed Kepler multi-exoplanetary systems measured with TTVs are biasing the samples toward pairs near resonances, the peaks may be overrepresented, but one can still find this statistical clustering feature by including all pairs of Kepler candidates (Goldreich \& Schlichting 2014). The excess near the 3:2 MMR is mostly associated with lowmass-planet systems, whereas that near the 2:1 MMR contains both low-mass-planet and gas-giant systems. If these systems formed through resonant capture during planetary migration, it appears that their asymptotic MMR is a sensitive function of planet mass, orbital characteristics, and relative migration rates (Ogihara \& Kobayashi 2013). Gas-giant pairs such as GJ876 (Laugh- 


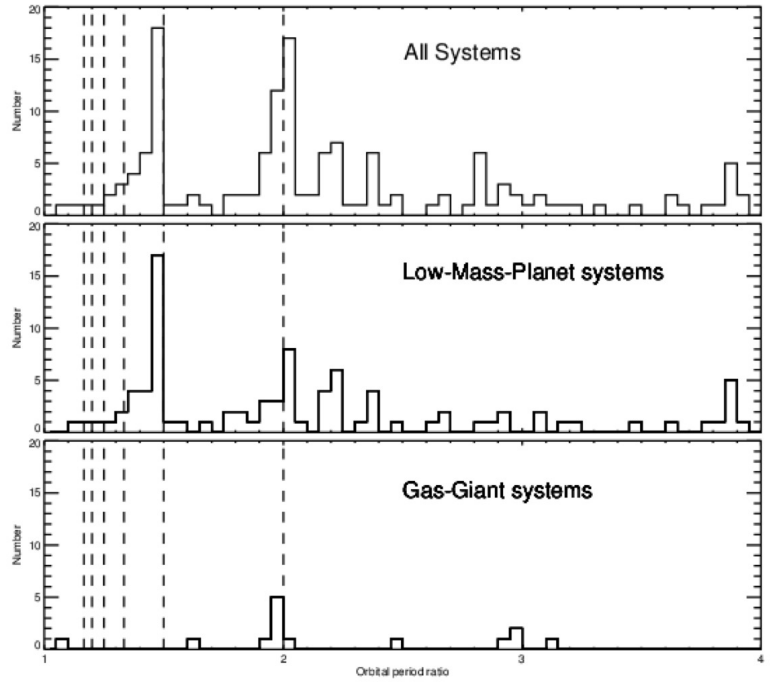

FIG. 1.- Top-bottom: distribution of period ratios of adjacent pairs from all the confirmed multiple-planet systems (top), just the low-mass-planet systems with both planet masses $\leq 30 M_{\oplus}$ (middle), and just the gas-giant systems with both planet masses $\geq 0.8 M_{\text {Jup }}$ (bottom). The vertical dashed lines indicate the firstorder MMR at 2:1, 3:2, 4:3, 5:4, 6:5 and 7:6, from right to left respectively. Note that only pairs with a period ratio $\leq 4$ are shown, which is about $70 \%$ of all planet pairs in the 157 confirmed systems. Whereas most of the gas-giant systems do not have MMRs smaller than 2:1, low-mass-planet systems can have MMRs down to $4: 3$, but there is a clear deficit of systems with a period ratio less than 4:3.

lin et al. 2001; Rivera \& Lissauer 2001; Laughlin et al. 2005) and HD82943 (Lee et al. 2006; Tan et al. 2013) cluster mostly around the 2:1 MMR because the Type II migration rate is relatively slow compared with the libration timescale for the 2:1 MMR.

However, Type I migration is considerably faster than Type II migration (Lin \& Papaloizou 1986). This allows migrating pairs to bypass wider resonances, with slower libration timescales (Murray \& Dermott 1999), and end up in more tightly packed configurations. It is noteworthy that there is a deficit of both low-mass-planet and gas-giant pairs with period ratios smaller than 4:3. Most Kepler planetary candidates have orbital periods within a few months, and the possibility of not detecting a transiting companion with similar periods is small. Thus, the observed deficit of closely packed resonant pairs and the preferential concentration of lower-mass pairs near the 3:2 MMR relative to the 2:1 MMR are statistically significant.

\section{METHOD}

In order to reproduce these observations in terms of the resonant-capture scenario, we use a two-dimensional (2D) module of the LA-COMASS package developed at Los Alamos to investigate the orbital evolution of multiple planets in a protostellar disk. This 2D hydrodynamical polar grid code solves the continuity and isothermal Navier-Stokes equations for gas inside a quasiKeplerian disk subject to the gravity of a one-solar-mass central star. The motion of multiple planets is calculated with a fourth-order Runge-Kutta solver. The evanescent boundary condition has been implemented in the code to provide wave killing zones at each edge of the disk (de Val-Borro et al. 2006). This code has been used exten- sively for planet-disk interaction studies (e.g., Li et al. (2009)).

In our simulations, the $2 \mathrm{D}$ disk is modeled within the radial range of $\left[0.3 R_{0}, 3.3 R_{0}\right]$ where $R_{0}$ is the distance unit in the code. We investigate two main configurations: a disk with multiple gas giants and a disk with multiple $10 M_{\oplus}$ planets. For the migration of gas giants, since their Type II migration does not sensitively depend on the disk structure (Lin \& Papaloizou 1986), we adopt models with smooth density, temperature and viscosity profiles. However, the pace and direction of the Type I migration does depend sensitively on the disk properties and they may be trapped in a disk near the magnetospheric truncation radius, the inner edge of "dead zone" or other locations such as the boundary between the viscous heating and irradiation heating region (Kretke \& Lin 2012).

For the migration of low-mass planets, we adopt a twozone disk model based on the $\alpha$ prescription for viscosity. In order to approximate a disk structure in which the magnetohydrodynamic turbulence is prevalent throughout the inner region and the mostly neutral outer regions with a "dead" midplane zone, we adopt a high value of $\alpha_{\text {vis }}=0.004$ for disk radius $a<a_{\text {crit }}=0.6 R_{0}$ and $\alpha_{v i s}=0.001$ for $a>a_{\text {crit }}$. Typical values of $R_{0}$ may range from a few stellar radii (for the inner disk boundary or the inner boundary of the dead zone) to a few AUs (for the interface between the viscously heated and irradiated disk regions). In a steady state (Kretke et al. 2009), this prescription leads to a disk surface density $(\Sigma)$ and pressure enhancement across $a_{\text {crit }}$. This $\Sigma$ distribution affects the direction and pace of low-mass-planets Type I migration (Paardekooper et al. 2010, 2011). The planets are placed relatively far away from $R_{0}$ so that their initial migration follows the typical Type I migration.

The ratio of disk scale height over disk radius is taken to be a constant of $h / r=0.05$. For all simulations, the initial semi-axis of planet pairs are all beyond $R_{0}$. The planets are held on their initial orbits for 1000 Keplerian periods and are released after their disk has adjusted to their perturbation. Our simulated time span is much shorter than the Myr disk depletion timescale. The overall change in the disk mass is modest.

\section{SIMULATION RESULTS}

The top panel of Figure 2 shows the evolution of the period ratio of two gas giants under a combination of two different mass ratios and two different sets of initial radial separations, based on the assumption that gas giants are formed independently and separated by modest period ratios $(\sim 3)$. Since the density waves excited by the two gas giants propagate throughout the remaining ring between them, gas diffuses through the gaps around each planet, in contrast to the shepherded planetary rings. After gas in the ring is depleted, the exchange of angular momentum between planets and inner/outer disks induces them to converge (Bryden et al. 2000). After a few thousand orbits, these gas giants capture each other into a 2:1 MMR. For the gas-giants pairs, we only simulated the convergent migration using a typical set of disk conditions. Since the Type II migration timescale is comparable to or even longer than the viscous evolution timescale of the disk, the gas giants can hardly pass through the 2:1 MMR location unless they are in a disk 

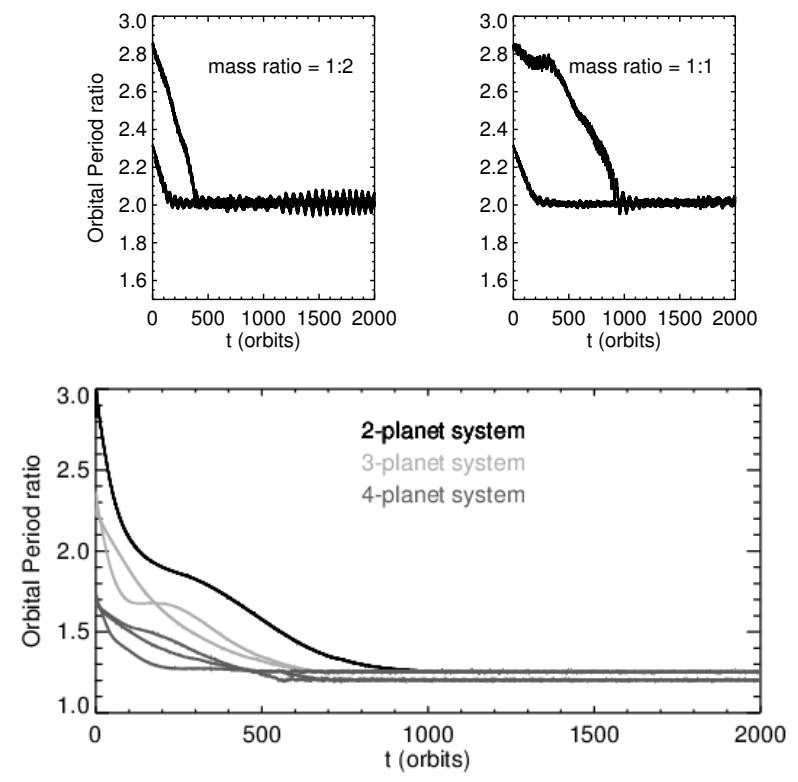

FIG. 2.- Top: the curves represent the period ratio evolution of gas-giant pairs with combinations of outer-to-inner planet mass ratios of 0.5 (left panel) and 1 (right panel), and initial period ratio of 2.83 and 2.31. All planets are released initially with circular orbits. The inner planet mass is fixed at $2 M_{J}$. They all settle to a period ratio of around 2:1. Bottom: the period ratio evolution of low-mass-planet pairs in two-planet (black curve), three-planet (light gray curves) and four-planet (dark gray curves) systems. All planets have $10 M_{\oplus}$. They all settle to a period ratio of around 5:4 or $6: 5$.

with extremely high accretion rate.

We also simulated the evolution of initially compact gas-giant pairs (with a period ratio less than 2). Population synthesis models (Ida et al. 2013) indicate that multiple gas-giant systems can form with relatively compact orbits because their progenitor embryos are separated by $\sim 10$ Hill's radii before they acquire sufficient mass to accrete gas efficiently. Our extensive simulations show that the orbits of these compact gas-giant pairs are not stable against their intense gravitational perturbation on each other (Zhou et al. 2007). In all cases, one of the two gas giants is scattered to large distances from its initial location. In the inner regions, the scattered planets may resume their migration. If they are able to catch up with their companions, they would eventually settle into the $2: 1$ resonance. The gas-giants' resonance "barrier" at the 2:1 MMR is confirmed by these simulations.

Typically the Type I migration timescale of a planet on a circular orbit is $\tau_{0} \sim h^{2} q^{-1} M_{\star} /\left(\Sigma_{p} R_{p}^{2}\right) \Omega_{p}^{-1}$, where $h$ is the aspect ratio of the disk, $q$ is the mass ratio of planet to star, $R_{p}$ is the location of planets and $\Sigma_{p}$ is the local surface density at $R_{p}$. The migration rate also sensitively dependent on the gradient of the surface density and temperature of the disk (Paardekooper et al. 2010, 2011). In this Letter, we only control the migration rate by applying different surface densities. Our disk model leads to the convergent evolution between adjacent pairs of two, three, or four low-mass-planet systems. In these simulations, the mass of each planet is assumed to be $10 M_{\odot}$ and the gas mass within the computational domain is $2 \times 10^{-3}$ that of the host star. The asymptotic period ratio of all neighboring pairs is around 5:4 or smaller. This ability to closely pack multiple low-mass-planet systems is due to their relatively rapid converging speed.

Such compact systems are rarely found among the Kepler planetary candidates. This apparent discrepancy between observations and simulations can be reduced considerably with much slower converging Type I migration rates. In order to illustrate this conjecture, we have performed a large set of simulations of multiple low-mass systems for a broad range of disk surface densities.

In these simulations, we have chosen the central star mass $M_{\star}=1 M_{\odot}, h / r=0.05$ and $\alpha_{v i s}=0.004 / 0.001$ for $a$ interior/exterior to $a_{c r i t}$. In a steady state, the disk accretion rate is given by $\dot{M}=1.5 \times 10^{-4} \cdot f$. $\left(\frac{1 A U}{R_{0}}\right)^{1.5} M_{\odot} / y r$, where $f=\frac{\Sigma_{0} R_{0}^{2}}{M_{\star}}$ is the disk-to-star mass ratio and $\Sigma_{0}$ is the disk surface density (in $\mathrm{g} / \mathrm{cm}^{2}$ ) at $R_{0}$. The transition radius $a_{\text {crit }}=0.6 R_{0}$ is also dependent on the disk accretion rate (Kretke et al. 2009) as $a_{\text {crit }} \propto \dot{M}^{0.5}$. Both the disk surface density normalization and the trapping location (associated with $R_{0}$ ) decrease as the disk accretion rate diminishes during disk depletion. For example, with $f=0.002$, we have $\dot{M}=3.6 \times 10^{-8} M_{\odot} / y r$ and $a_{c r i t}=2.5 A U$. In our simulations, there is no time evolution of the disk, except for one case shown in Figure 4. In other cases, the accretion rate is constant along the simulations. Varying $\dot{M}$ is equivalent to varying disk mass which is equivalent to varying the establishing time of the planet orbital architecture.

From these simulations, we find that the asymptotic period ratios between adjacent pairs decrease toward unity as the accretion rate increases. Figure 3 shows a general trend that lower disk accretion rates lead to relatively wider spacing for low-mass-planet systems. These results suggest that the accretion rate must be $<2 \cdot 10^{-8} M_{\odot} / y r$ to reproduce the observed paucity. If this paucity is a signature of some alternative dynamical process, like instability, then we can constrain the forming stage of the planetary systems from the accretion rate corresponding to the final period ratio larger than the 4:3 MMR. The corresponding disk-to-star mass ratio in this region is $<6.7 \times 10^{-4}$. The reproduction of the observed enhancement of adjacent low-mass-planet pairs with 3:2 MMR requires even lower disk accretion rates $\left(\dot{M}<10^{-8} M_{\odot} / y r\right)$ especially in systems with more than two planets. Note that the inferred accretion rates around classical T Tauri stars in the Taurus and Ophiuchus complex (Natta et al. 2006) range mostly between $10^{-8}-10^{-7} M_{\odot} y r^{-1}$.

In order to extract constraints on the disk accretion rate from Figure 1, we need to verify that the stability of compact systems may be preserved (Gladman 1993). Systematic studies (Zhou et al. 2007) show that in a gas-free environment, multiple equal-mass low-massplanet systems with initially circular orbits separated by $k_{0}=\Delta a / R_{H}<10\left(\right.$ where $\left.R_{H}=\left(2 M_{p} / 3 M_{*}\right)^{1 / 3} a\right)$ become dynamically unstable over the Gyr main sequence lifespan of the host stars. For non-resonant $M_{p}=10 M_{\oplus}$ low-mass-planet systems, this stability criterion requires the period ratio to be larger than $\sim 1.43$, which could account for the paucity of multiple systems with a period ratio between adjacent planets smaller than that of the 3:2 MMR. Multiple gas-giant systems with a period ratio between adjacent planets smaller than that of the 


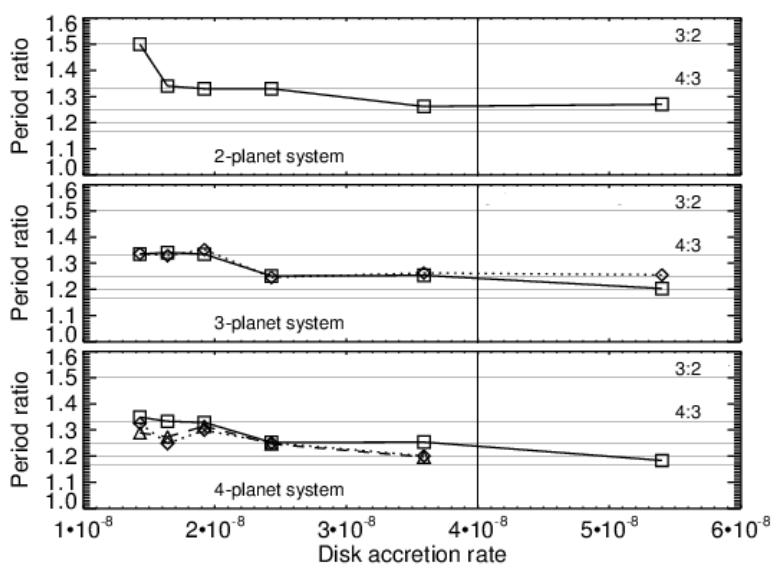

FIG. 3.- Panels from top to bottom show the final period ratio of adjacent pairs in multiple low-mass-planet systems with two, three and four planets of $10 M_{\oplus}$ each. The horizontal lines indicate the first-order MMRs at 3:2, 4:3, 5:4, 6:5 and 7:6. The vertical line marks the observed disk accretion rate for a typical young solar mass system with an age $\sim 1$ Myr (Natta et al. 2006).

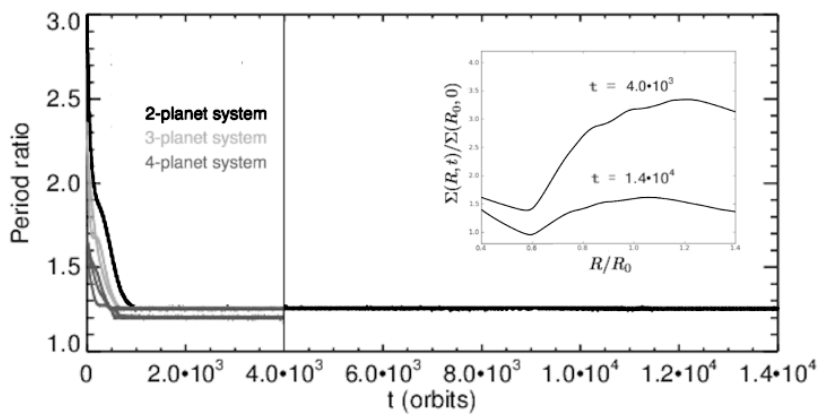

FIG. 4.- Pairs of low-mass-planets undergo Type I migration and capture each other within $10^{3}$ orbits, as indicated by the left panel. Continued simulations up to $10^{4}$ orbits of such systems demonstrate that the pair remains around the 5:4 MMR. A gradual decrease of the disk surface density is implemented, as shown in the insert.

\section{2:1 MMR are also unstable on Gyr timescale.}

However, the stability of systems with near MMRs may be better preserved against long-term dynamical instability. To illustrate, we select a pair of low-mass planets that are captured into each other's 5:4 MMR in a disk initially with $f=0.002$. Such a system corresponds to $k_{0}=6$. If it is out of resonance, it would become dynamically unstable in $<10^{3}$ orbits in the absence of gas and $<10^{4}$ orbits if it is embedded in a minimum mass solar nebula. We extend our simulation for an additional $10^{4}$ orbits while the disk surface density is prescribed to decrease exponentially over that timescale. The results in Figure 4 indicate that once the low-mass-planet pairs are captured into a tight MMR, they tend to remain in these MMRs in the absence of major perturbations before the depletion of the disk. However, the later longterm orbital evolution of planets with an absence of gas may break the system. Although we can not exclude the instability criterion as an contribution to the paucity of a period ratio smaller than the 4:3 MMR, we can take this as a clue to the formation stage. If instability is responsible for the paucity, it might indicate that the low-mass-planet pairs with a period ratio larger than 4:3 formed in a somewhat late stage of the disk.

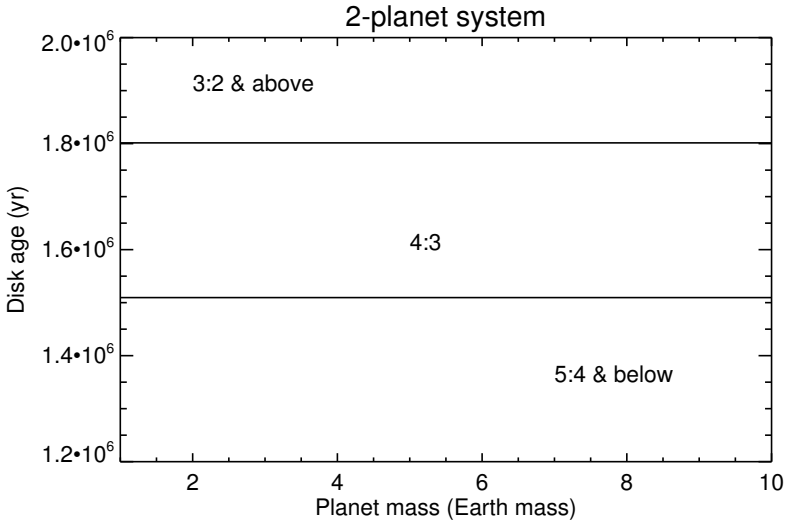

Fig. 5.- Two solid lines, from top to bottom, mark the earliest formation time for planet pairs that produce final period ratios of $3: 2$ and $4: 3$, respectively. An exponentially decreasing disk accretion rate model is used in this calculation.

Based on the results from Figures 3 and 4, we infer that, low-mass-planet systems can capture each other into 4:3 and/or 3:2 MMR,s provided they undergo Type I migration when the accretion rate $(\dot{M})$ or the mass of their natal disks is relatively low. The results in Figure 3 indicate that in order for a pair of $10 M_{\oplus}$ lowmass planets to attain an asymptotic period ratios of $4: 3$ and $3: 2$, the upper limits of the disk-to-star mass ratios are at $f=6.7 \times 10^{-4}$ and $f=4 \times 10^{-4}$, respectively. Since the Type I migration rate is linearly proportional to the product of $q$ and $f$, low-mass-planet pairs would approach each other's 4:3 (or 3:2) MMRs with the same critical relative speed if their $q \cdot f \sim 2 \times 10^{-8}$ (or $\sim 1.2 \times 10^{-8}$ ). The condition for MMR capture requires that the migration timescale through the characteristic width $\left(\tau_{m i g} \sim \Delta a / \dot{a}\right)$ is longer than the libration timescale $\left(\tau_{l i b}\right)$. If we take into account that the libration time of the lowest-order MMR is $\propto q^{-1 / 2}$ and $\Delta a \propto q^{1 / 2}$ in the first order of the expansion (Murray \& Dermott 1999), the critical condition for the lowest-order of MMR capture would be roughly independent on the planet-tostar mass ratio.

In order to quantify the stage of the disk according to the accretion rate, we use the observations of a relatively young $\left(t_{a} \sim 1 \mathrm{Myr}\right)$ star forming region, $\rho$ Oph (Natta et al. 2006), where the accretion rate around $\sim 1 M_{\odot}$ star is about $4 \times 10^{-8} M_{\odot} / y r$. For $R_{0}=4.1 A U$, the corresponding value $f=0.002$. The accretion rate is also observed to decline over a timescale of 3-5 Myr. We introduce an approximation for the disk accretion rate $\dot{M}=4 \times 10^{-8} \cdot e^{-\left(\frac{t-t_{a}}{\tau_{D}}\right)} M_{\odot} / y r$ for $t \geq t_{a}$, where $t$ indicates the age of the disk, $t_{a} \sim 1 M y r$, and $\tau_{D}$ is the disk lifetime. Figure 5 shows the dependency of final period ratios on the planet masses and the MMRs formation times. It confirms that a relatively large period ratio (such as 3:2) requires the low-mass-planet pairs to have migrated and captured each other late in the disk evolution stage when the disk surface density was sufficiently depleted.

\section{SUMMARY}

These simulation results for multiple gas-giant and lowmass-planet systems place important constraints on the planet formation stage with respect to the disk evolution. 
According to the core accretion scenario, the formation of gas giants must be preceded by the emergence of sufficiently massive $\left(>10 M_{\oplus}\right)$ protostellar embryos in a gasrich environment, presumably during the early stage of disk evolution. These cores, if retained in a dense disk, would either congregate and effectively merge near some trapping radius to become cores of proto-gas-giant planets or be mostly scattered into or far from their host stars. A significant "left-over" population would have produced compact pairs with small period ratios that are not consistent with observations. Around stars that only bear relatively low-mass planets, their dynamical configuration may be established during the advanced stages of disk evolution when the disk gas is severely depleted. It is also possible that these low-mass planets were assembled over several millions of years.

We thank the referee, Frederic Rasio, for helpful comments that improved the manuscript. We acknowledge support from the LDRD program and IGPP of Los Alamos National Laboratory. H.L. and D.N.C.L. also acknowledge support from the UC-fee program of University of California. Simulations were carried out using the Institutional Computing resources at LANL.

\section{REFERENCES}

Batalha, N. M., Rowe, J. F., Bryson, S. T., et al. 2013, ApJS, 204, 24

Bryden, G., Różyczka, M., Lin, D. N. C., et al. 2000, ApJ, 540, 1091

de Val-Borro, M., Edgar, R. G., Artymowicz, P., et al. 2006 , MNRAS, 370, 529

Fabrycky, D. C., \& Kepler Science Team. 2012, in AAS/Division of Dynamical Astronomy Meeting, Vol. 43, AAS/Division of Dynamical Astronomy Meeting, 01.03

Gladman, B. 1993, Icarus, 106, 247

Goldreich, P. 1965, MNRAS, 130, 159

Goldreich, P., \& Schlichting, H. E. 2014, AJ, 147, 32

Goldreich, P., \& Tremaine, S. 1980, ApJ, 241, 425

Henrard, J. 1982, Celestial Mechanics, 27, 3

Ida, S., Lin, D. N. C., \& Nagasawa, M. 2013, ApJ, 775, 42

Kley, W. 2000, MNRAS, 313, L47

Kley, W., Peitz, J., \& Bryden, G. 2004, A\&A, 414, 735

Kretke, K. A., \& Lin, D. N. C. 2012, ApJ, 755, 74

Kretke, K. A., Lin, D. N. C., Garaud, P., et al. 2009, ApJ, 690, 407

Laughlin, G., Butler, R. P., Fischer, D. A., et al. 2005, ApJ, 622, 1182

Laughlin, G., Chambers, J., \& Fischer, D. 2001, in Bulletin of the American Astronomical Society, Vol. 33, American Astronomical Society Meeting Abstracts, 1304

Lee, M. H., Butler, R. P., Fischer, D. A., et al. 2006, ApJ, 641, 1178

Lee, M. H., Fabrycky, D., \& Lin, D. N. C. 2013, ApJ, 774, 52

Lee, M. H., \& Peale, S. J. 2002a, ApJ, 567, 596

-. 2002b, ArXiv Astrophysics e-prints, astro-ph/0209176
Li, H., Lubow, S. H., Li, S., et al. 2009, ApJL, 690, L52

Lin, D. N. C., Bodenheimer, P., \& Richardson, D. C. 1996, Nature, 380, 606

Lin, D. N. C., \& Papaloizou, J. 1986, ApJ, 309, 846

Lissauer, J. J., Fabrycky, D. C., Ford, E. B., et al. 2011a, Nature, 470,53

Lissauer, J. J., Ragozzine, D., Fabrycky, D. C., et al. 2011b, ApJS, 197, 8

Lissauer, J. J., Marcy, G. W., Rowe, J. F., et al. 2012, ApJ, 750, 112

Mayor, M., Udry, S., Lovis, C., et al. 2009, Astron. Astrophys., 493, 639

Murray, C. D., \& Dermott, S. F. 1999, Solar system dynamics

Natta, A., Testi, L., \& Randich, S. 2006, A\&A, 452, 245

Ogihara, M., Duncan, M. J., \& Ida, S. 2010, ApJ, 721, 1184

Ogihara, M., \& Ida, S. 2009, in AAS/Division for Planetary

Sciences Meeting Abstracts, Vol. 41, AAS/Division for

Planetary Sciences Meeting Abstracts \#41, 40.05

Ogihara, M., \& Kobayashi, H. 2013, ApJ, 775, 34

Paardekooper, S.-J., Baruteau, C., Crida, A., et al. 2010, MNRAS, 401, 1950

Paardekooper, S.-J., Baruteau, C., \& Kley, W. 2011, MNRAS, 410, 293

Rivera, E. J., \& Lissauer, J. J. 2001, ApJ, 558, 392

Schlaufman, K. C., Lin, D. N. C., \& Ida, S. 2010, ApJL, 724, L53

Tan, X., Payne, M. J., Lee, M. H., et al. 2013, ApJ, 777, 101

Ward, W. R. 1997, ApJL, 482, L211

Weidenschilling, S. J., \& Davis, D. R. 1985, Icarus, 62, 16

Wisdom, J. 1980, AJ, 85, 1122

Zhou, J.-L., Lin, D. N. C., \& Sun, Y.-S. 2007, ApJ, 666, 423 